\title{
Dexamethasone in patients with acute lung injury from acute monocytic leukaemia
}

\author{
É. Azoulay*, E. Canet*, E. Raffoux*, E. Lengliné\#, V. Lemiale*, F. Vincent*, \\ A. de Labarthe ${ }^{\#}$, A. Seguin*, N. Boissel ${ }^{\#}$, H. Dombret ${ }^{\#}$ and B. Schlemmer*
}

ABSTRACT: The use of steroids is not required in myeloid malignancies and remains controversial in patients with acute lung injury (ALI) or acute respiratory distress syndrome (ARDS). We sought to evaluate dexamethasone in patients with ALI/ARDS caused by acute monocytic leukaemia (AML FAB-M5) via either leukostasis or leukaemic infiltration.

Dexamethasone (10 mg every $6 \mathrm{~h}$ until neutropenia) was added to chemotherapy and intensive care unit (ICU) management in 20 consecutive patients between 2005 and 2008, whose data were compared with those from 20 historical controls (1994-2002). ICU mortality was the primary criterion. We also compared respiratory deterioration rates, need for ventilation and nosocomial infections.

$17(85 \%)$ patients had hyperleukocytosis, 19 (95\%) had leukaemic masses, and all 20 had severe pancytopenia. All patients presented with respiratory symptoms and pulmonary infiltrates prior to AML FAB-M5 diagnosis. Compared with historical controls, dexamethasone-treated patients had a significantly lower ICU mortality rate $(20 \%$ versus $50 \% ; p=0.04)$ and a trend for less respiratory deterioration $(50 \%$ versus $80 \% ; p=0.07)$. There were no significant increases in the rates of infections with dexamethasone.

In conclusion, in patients with ALI/ARDS related to AML FAB-M5, adding dexamethasone to conventional chemotherapy seemed effective and safe. These results warrant a controlled trial of dexamethasone versus placebo in AML FAB-M5 patients with noninfectious pulmonary infiltrates.

KEYWORDS: Acute lung injury, acute monocytic leukaemia, acute respiratory distress syndrome, dexamethasone, glucocorticoids

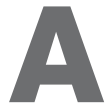
mong patients with acute leukaemia, up to half experience respiratory events early in the course of the disease $[1,2]$. In this situation, progression to acute respiratory failure requiring ventilatory support is a severe complication that is not only frequently fatal $[3,4]$, but also delays the administration of optimal chemotherapy [5].

In patients with acute leukaemia and pulmonary infiltrates, infection must be sought and treated empirically [3]. However, pulmonary involvement may be directly due to the malignancy. In patients with myelomonocytic or monocytic acute leukaemia, leukaemia-related pulmonary involvement is frequent and severe [6-8]. The diagnosis rests on negative findings from extensive tests for the main infectious and noninfectious causes. Leukaemiarelated pulmonary involvement includes pulmonary leukostasis, leukaemic pulmonary infiltrates and lysis pneumopathy [9-18]. We previously described 20 patients with acute monocytic leukaemia (AML; French-American-British (FAB) classification M5) who presented with either leukaemic infiltrates or leukostasis at the earliest phase of the malignancy [8]. All patients experienced lysis pneumopathy within hours of chemotherapy initiation. Mechanical ventilation and mortality rates were high, indicating a need to develop better treatment strategies. In addition to early intensive care unit (ICU) management, best supportive care, and rapid cytoreduction via hydration and chemotherapy, anti-inflammatory therapy would be expected to improve outcomes in these patients.

Steroid therapy has been used with variable results to prevent acute respiratory distress syndrome (ARDS) in high-risk patients [19], in short courses to treat early severe ARDS [20], and as rescue therapy in patients with persistent ARDS [21, 22]. In AML FAB-M5 patients, steroids would be expected to decrease cytokine and oxidant release, blast adhesion to endothelial cells and blast degeneration within the interstitium [15]. Steroids may both limit the extent of initial lung injury, as shown in patients with all-trans-retinoic acid (ATRA) syndrome [23], and prevent lysis pneumopathy.
AFFILIATIONS

${ }^{*}$ Medical ICU and

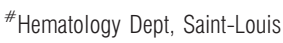
Hospital, Paris, France.

CORRESPONDENCE

É. Azoulay

AP-HP

Hôpital Saint-Louis

Medical ICU

75010 Paris

France

E-mail: elie.azoulay@sıs.ap-hopparis.fr

Received:

May 242011

Accepted after revision:

July 012011

First published online:

Aug 042011 
The objective of this study was to evaluate whether adding dexamethasone to the chemotherapy protocol in patients with AML FAB-M5 and acute lung injury (ALI)/ARDS due to leukaemic pulmonary involvement improved survival and decreased the incidence of respiratory deterioration and the need for ventilatory support.

\section{PATIENTS AND METHODS}

Consecutive patients with newly diagnosed and previously untreated AML FAB-M5 admitted to our ICU between January 1, 2005 and December 31, 2008 for acute respiratory failure were eligible for enrolment. The main inclusion criteria were as follows: 1) cytologically documented AML FAB-M5 (according to the World Health Organization classification, $\geqslant 20 \%$ leukaemic blasts in the bone marrow with monocytic cells comprising $>80 \%$ of nonerythroids cells); 2) respiratory symptoms and pulmonary infiltrates at the earliest phase of AML FAB-M5; 3) ICU admission for acute respiratory failure defined by respiratory rate $>30$, oxygen saturation $<90 \%$ or signs of respiratory distress; 4) no chemotherapy before ICU admission; and 5) no clinical or microbiological evidence of infection. Our institutional review board (Saint-Louis Teaching Hospital, Paris, France) approved the prospective data collection of AML cases, which was conducted in accordance with the Declaration of Helsinki, International Conference on Harmonization, and Guidelines for Good Clinical Practice. The protocol for treating AML FAB-M5 patients with noninfectious pulmonary manifestations at the earliest phase of the disease was amended by the addition of dexamethasone. All patients were informed of the diagnosis of AML FAB-M5, the likelihood that the pulmonary manifestations were related to the leukaemia, and the use of dexamethasone added to conventional chemotherapy to limit the severity of ALI/ARDS severity and to prevent lysis pneumopathy.

ICU management was provided jointly by intensivists and haematologists. All patients underwent induction chemotherapy with an anthracycline plus cytarabine-based regimen. Based on previous experience in hyperleukocytic patients with acute promyelocytic leukaemia, dexamethasone has been preferred to methylprednisolone or hydrocortisone [24]. Dexamethasone (10 mg every $6 \mathrm{~h}$ i.v.) was given until leukopenia $\left(<11 \times 10^{9} \cdot \mathrm{L}^{-1}\right)$ occurred. ICU management included optimal ventilatory support with supplemental oxygen, noninvasive ventilation, and/or invasive mechanical ventilation as appropriate [25], and other life-sustaining therapies as required [8, 26]. All patients underwent noninvasive tests for pathogens in sputum, induced sputum, nasopharyngeal aspirates, blood and urine $[25,27]$. Echocardiography was normal in all patients. Patients requiring endotracheal intubation routinely underwent bronchoscopy and bronchoalveolar lavage (BAL). Antibiotics covering community-acquired pathogens (third-generation cephalosporin or piperacillin-tazobactam plus a macrolide/ quinolone) were given to all patients for 7 days, despite negative results for infection from all tests.

Variables listed in tables 1 and 2 were collected prospectively. Vital status at ICU and hospital discharge was known for all study patients.

Safety evaluation included mostly the proportion of patients presenting with hospital-acquired bacterial or opportunistic infection.

\section{Statistical analysis}

Quantitative parameters are reported as median (interquartile range; 25th-75th percentiles) and qualitative parameters as number and percentage. Comparisons were performed between the 20 patients given dexamethasone and 20 historical controls (previously published) who received the same treatment without steroids. ICU mortality was the primary evaluation criterion. Categorical variables were compared using the Chi-squared test or Fisher's exact test, as appropriate. Continuous variables were compared using the Mann-Whitney U-test or the Wilcoxon test, as appropriate.

Associations between patient characteristics and hospital mortality were assessed using a logistic regression model. Multivariable analysis was performed using stepwise forward selection to introduce variables whose $p$-values were $<0.20$ by univariate analysis. Then, the absence of a significant increase in the likelihood value after omission of each of the remaining variables was checked. Goodness of fit was evaluated using the HosmerLemeshow statistic. Odds ratios and their 95\% confidence intervals were computed. $p$-values $<0.05$ were considered statistically significant. Analyses were done using the SAS 9.1 software package (SAS Institute, Cary, NC, USA).

\section{RESULTS}

From January 2005 to December 2008, 450 patients with newly diagnosed acute myeloid leukaemia were admitted to the SaintLouis Teaching Hospital, including 45 (10\%) with AML FAB-M5, of whom 20 (44\%) required ICU admission before chemotherapy initiation for respiratory manifestations with onset before the diagnosis of leukaemia (fig. 1). Of these 20 patients, 11 were admitted directly to the ICU for acute respiratory failure and nine were transferred from the haematological wards within 12 (6-36) $\mathrm{h}$ of admission. In 14 patients, AML FAB-M5 was diagnosed during the ICU stay.

As reported in table 1, there were 13 males and seven females with a median age of 42 (33-60) yrs. None had any comorbidity. All patients had severe acute respiratory failure with tachypnoea and profound hypoxaemia. The chest radiographs consistently showed diffuse lung infiltrates. The Simplified Acute Physiology Score version II score was 39 (29-50). All 20 patients were febrile and all but one had physical evidence of leukaemic masses. Laboratory findings included high white blood cell count in 17 patients, circulating blast cells in 16 and platelet count $<501 \times 10^{9}$ cells $\cdot \mathrm{L}^{-1}$ in 12 . The three patients without hyperleukocytosis had $<101 \times 10^{9}$ cells $\cdot \mathrm{L}^{-1}$ with no circulating blast cells. Disseminated intravascular coagulation was present in seven patients.

As shown in table 2, within a few hours after chemotherapy initiation, 10 patients had no respiratory status deterioration or increase in oxygen requirements and 10 had a deterioration in oxygenation requiring invasive mechanical ventilation. An additional patient required intubation and ventilation because of complex ventricular arrhythmia. Median length of ICU stay was 7 days (3.5-14). ICU and hospital survival rates were both $80 \%$ (four deaths). All 16 survivors were in remission after neutropenia recovery and received consolidation chemotherapy, and 1-yr survival was $60 \%$ (eight deaths).

None of the baseline characteristics differed significantly between the historical controls and the dexamethasone-treated 
TABLE 1 Patient characteristics at intensive care unit (ICU) admission

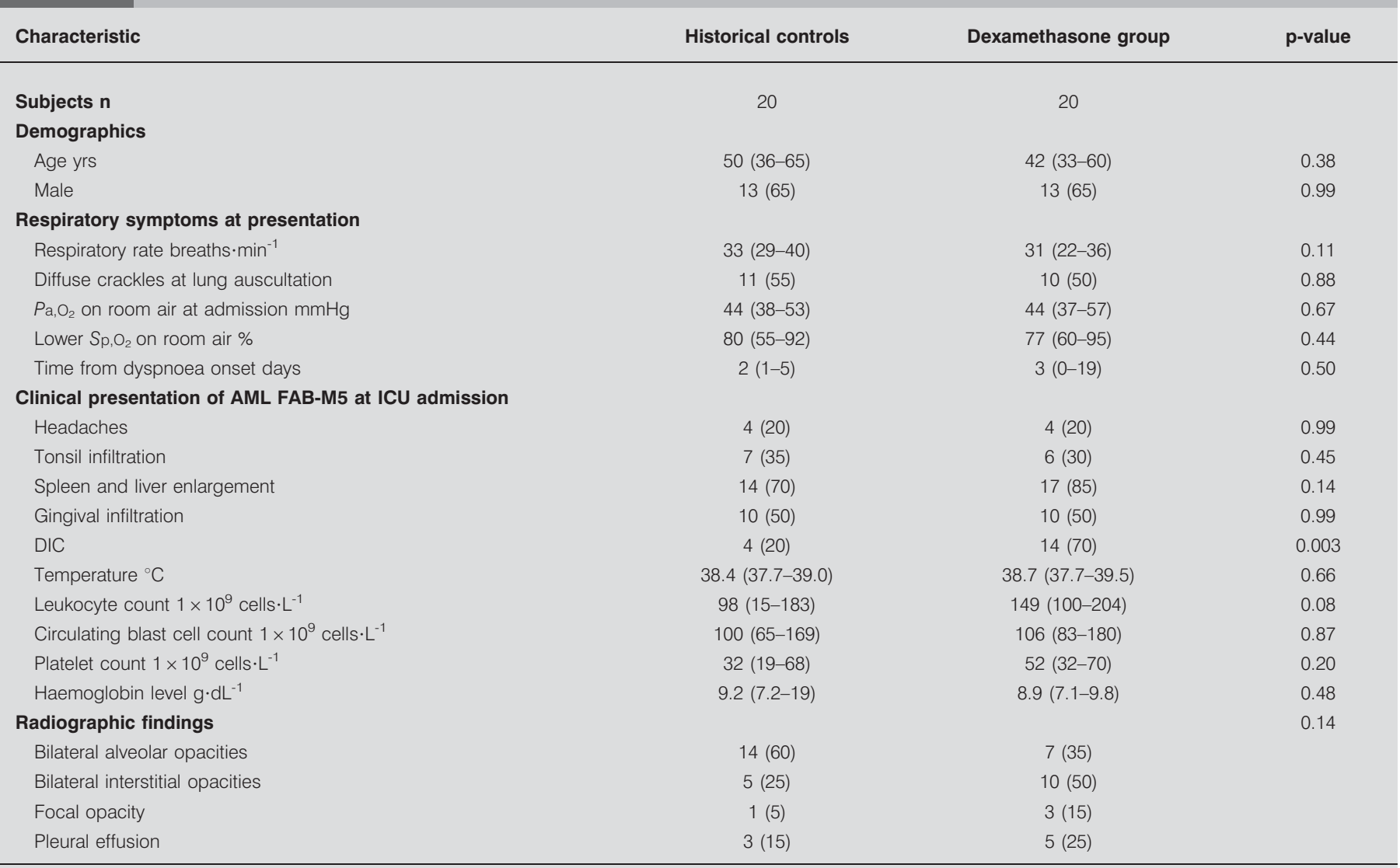

Data are presented as median (interquartile range) or $\mathrm{n}(\%)$, unless otherwise stated. $\mathrm{Pa}_{\mathrm{a}} \mathrm{O}_{2}$ : partial pressure of oxygen in arterial blood; $\mathrm{Sp}, \mathrm{O}_{2}$ : arterial oxygen saturation measured by pulse oximetry; AML FAB-M5: acute monocytic leukaemia French-American-British subtype 5; DIC: diffuse intravascular coagulation.

TABLE 2 Intensive care unit (ICU) management in historical control patients and in patients given dexamethasone

\begin{tabular}{lcc} 
& Historical controls & Dexamethasone group \\
\hline Subjects n & 20 & 20 \\
SAPS II at ICU admission & $44(35-51)$ & $39(29-50)$ \\
Time from hospital to ICU admission days & $1(0-3)$ & $0.5(0-3)$ \\
Bronchoscopy and BAL performed & $20(100)$ & $5(20)$ \\
Deterioration of respiratory status after chemotherapy initiation & 0.54 \\
$\quad$ None & $0(0)$ & $4(20)$ \\
Increased oxygen needs & $4(20)$ & $6(30)$ \\
$\quad$ Invasive mechanical ventilation & $15(75)$ & $10(50)$ \\
Use of NIV & $11(55)$ & $3(15)$ \\
Need for NIV or invasive MV & $15(75)$ & $11(55)$ \\
Cardiac arrest after chemotherapy initiation & $3(15)$ & $1(5)$ \\
ICU-acquired events & & 0.07 \\
$\quad$ Invasive fungal infection & $3(15)$ & 0.01 \\
$\quad$ Hospital-acquired infection & $7(35)$ & 0.18 \\
$\quad$ Septic shock & $11(55)$ & 0.01 \\
Length of ICU stay days & $8(3.5-18.5)$ & $2(10)$ \\
ICU mortality & $10(50)$ & $6(30)$ \\
\hline
\end{tabular}

Data are presented as median (interquartile range) or $\mathrm{n}(\%)$, unless otherwise stated. SAPS II: Simplified Acute Physiology Score version II; BAL: bronchoalveolar lavage; NIV: noninvasive mechanical ventilation; MV: mechanical ventilation. 


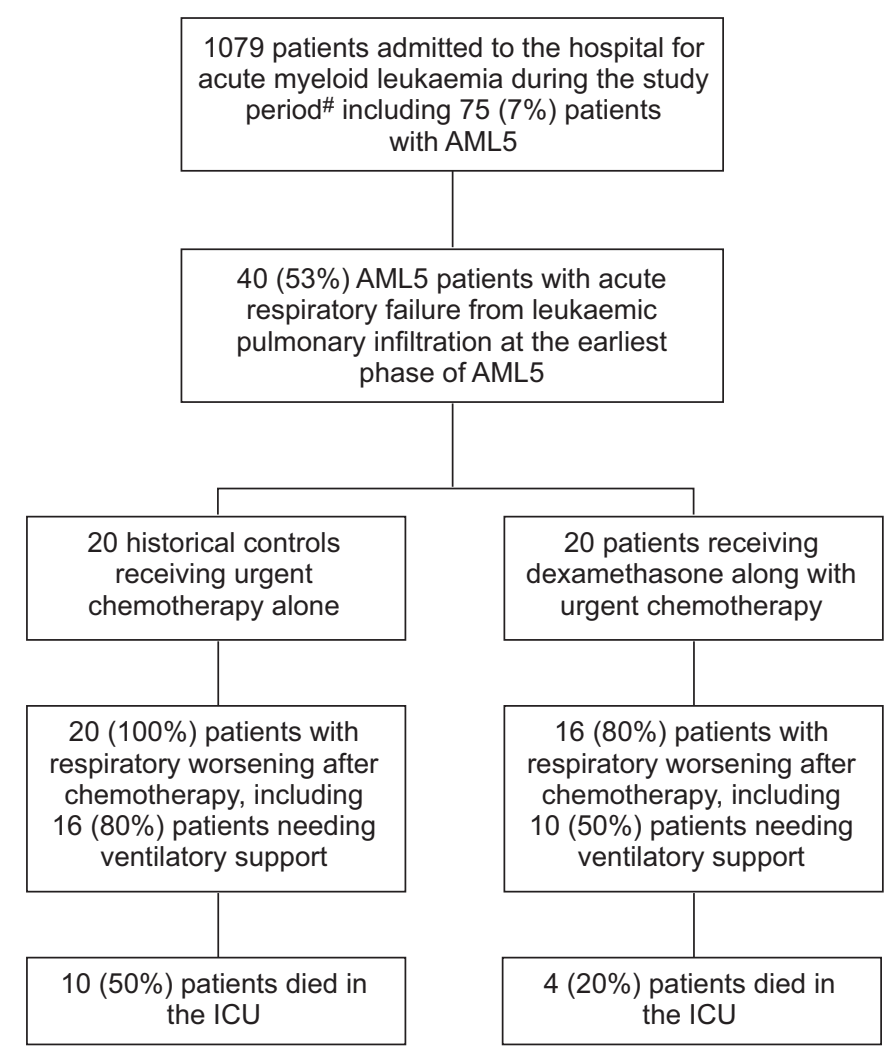

FIGURE 1. Patient flow chart. ICU: intensive care unit; AML5: acute myelocytic leukaemia FAB-M5. *: the study periods ran from January 1994 to July 2002 and from January 2005 to December 2008.

patients (tables 1 and 2). In particular, the severity of respiratory failure and characteristics of the leukaemia were well balanced between the two groups.

In-ICU mortality was significantly lower in the dexamethasone group than in the control group $(20 \%$ versus $50 \%$; $p=0.04)$. In the dexamethasone-treated group, we found decreases in the occurrence of respiratory status deterioration, need for increased oxygen flow and need for ventilatory support compared with the controls (fig. 2). Chemotherapy initiation was followed by respiratory status deterioration in only four $(20 \%)$ dexamethasonetreated patients compared with $20(100 \%)$ controls.

Dexamethasone therapy was not associated with increased rates of hospital-acquired bacterial or invasive fungal infections.

Table 3 reports the results of the multivariate analysis for factors associated with ICU mortality. Higher respiratory rate and lower arterial oxygen saturation measured by pulse oximetry at ICU admission were independently associated with ICU mortality. Dexamethasone therapy was associated with a trend for a decrease in ICU mortality by multivariate analysis.

\section{DISCUSSION}

We evaluated the efficacy and safety of dexamethasone therapy in patients with AML FAB-M5 and noninfectious pulmonary infiltrates. Compared with historical controls who did not receive dexamethasone, patients given dexamethasone had a decrease in the severity of pulmonary involvement and a lower rate of respiratory status deterioration after chemotherapy

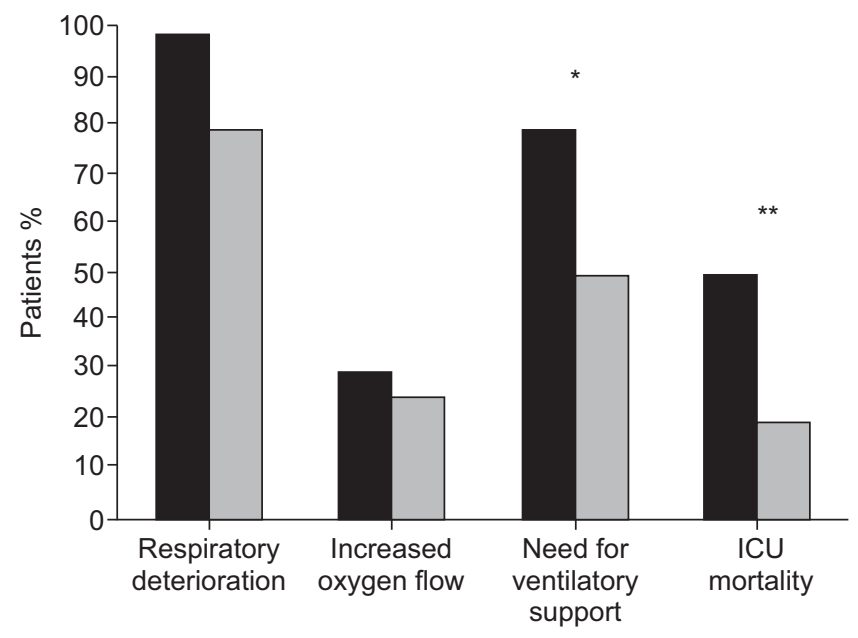

FIGURE 2. Impact of dexamethasone therapy on clinical outcomes. An additional patient from the dexamethasone group received ventilation for complex arrhythmia. $\mathbf{n}$ : dexamethasone-treated patients; $\mathbf{a}$ : historical controls. ICU: intensive care unit. ${ }^{*}: p<0.05 ;{ }^{*}: p<0.01$.

initiation. ICU mortality was lower in the dexamethasonetreated patients than in the historical controls.

Pulmonary leukostasis occurs in patients with acute myeloid leukaemia and rapidly increasing white blood cell count $[10,11]$, and is consistently present when the count exceeds $2001 \times 10^{9}$ cells $\cdot \mathrm{L}^{-1}$ [12]. Pulmonary leukostasis leads to endothelial injury and activation from microvascular invasion, with hyperviscosity, leukocytic microthrombi, oxygen steal and hypoxia [13-15]. Leukaemic pulmonary infiltrates occur in patients with or without hyperleukocytosis. This fact suggests that both the type of the blasts and their affinity for the pulmonary endothelium may be responsible for lung injury $[8,28]$. Autopsy studies have shown blast aggregates within the vessel lumina [29]. The infiltrates typically follow the lymphatic routes along the bronchovascular bundles, interlobular septa and pleural interstitial tissue [16-18]. Finally, lysis pneumopathy occurs either immediately or early after chemotherapy initiation as a manifestation of acute tumour lysis syndrome. Lysis pneumopathy results in diffuse alveolar damage [30] and is most common in

\begin{tabular}{|c|c|c|c|}
\hline $\begin{array}{l}\text { Factors ir } \\
\text { care unit }\end{array}$ & $\begin{array}{l}\text { lependentl } \\
\text { cU) mortal }\end{array}$ & $\begin{array}{l}\text { ssociated with i } \\
\text { by multivariable }\end{array}$ & $\begin{array}{l}\text { ensive } \\
\text { nalysis }\end{array}$ \\
\hline & Odds ratio & $\begin{array}{l}\text { 95\% confidence } \\
\text { interval }\end{array}$ & $\mathrm{p}$-value \\
\hline $\begin{array}{l}\text { Respiratory rate at } \\
\text { admission per point }\end{array}$ & 1.22 & $1.01-1.48$ & 0.036 \\
\hline $\begin{array}{l}\text { Dexamethasone (versus } \\
\text { historical controls) }\end{array}$ & 0.12 & $0.01-1.23$ & 0.054 \\
\hline $\begin{array}{l}\text { Time from hospital to } \\
\text { ICU admission per day }\end{array}$ & 1.17 & $0.97-1.40$ & 0.098 \\
\hline $\begin{array}{l}\text { SAPS II score at ICU } \\
\text { admission per point }\end{array}$ & 1.06 & $0.96-1.17$ & 0.216 \\
\hline
\end{tabular}


patients with hyperleukocytic acute myeloid leukaemia, particularly the myelomonocytic subtypes with abnormal marrow eosinophils [31].

Steroids have been used in early severe ARDS to improve oxygenation by decreasing lung collagen and oedema formation $[32,33]$. However, steroid use in patients with persistent ARDS is controversial [21,22]. Steroids are a major component of the treatment regimen for acute lymphocytic leukaemia but are not used in patients with acute myeloid leukaemia [34]. In patients with promyelocytic leukaemia given ATRA, dexamethasone was very effective in preventing or treating ATRArelated pulmonary toxicity $[23,35]$.

Noninfectious pulmonary involvement is particularly common in patients with hyperleukocytotic myeloid leukaemia, particularly of the M4 and M5 subtypes [18]. In a previous study, we described 20 AML FAB-M5 patients with acute respiratory failure from pulmonary leukostasis and leukaemic infiltration before the diagnosis of leukaemia [8]. All 20 patients had post-chemotherapy lysis pneumopathy. 10 patients died, indicating a need for an intervention targeting the pathophysiological mechanisms responsible for the initial lung injury and subsequent respiratory status deterioration. Steroid therapy was a good candidate, as steroids are widely used in various subsets of ALI [33] and chemotherapy-related pulmonary toxicity [23]. Our data suggest that steroid therapy may not only limit pulmonary leukostasis and leukaemic infiltration, but also prevent lysis pneumopathy. Compared with the control group, the dexamethasone-treated group was characterised by lower rates of respiratory status deterioration, mechanical ventilation and death. We found no significant increase in infections in the dexamethasone-treated group compared with the control group.

Our study has several limitations. We used historical controls, and the recruitment period from the first control to the last dexamethasone-treated patient spans $15 \mathrm{yrs}$, during which time changes in the management of ALI/ARDS and increasing experience may have affected patient outcomes [36]. However, the dexamethasone and control patients were not different at baseline and received the same haematological and ICU management. Our data suggest that a multicentre randomised controlled trial testing the risk/benefit ratio of dexamethasone in patients with AML FAB-M5 and noninfectious pulmonary involvement may be warranted. Whether such a trial should be extended to all patients with acute leukaemia-related noninfectious pulmonary involvement requires discussion. A second limitation of the study is that we did not record noninfectious adverse effects of dexamethasone therapy (e.g. poor glucose control). Future studies will need to record all possible adverse effects. Last, we assumed that all patients with AML FAB-M5, acute respiratory failure, pulmonary infiltrates and negative tests for infection had leukaemia-related pulmonary involvement. This reflects our standard diagnostic strategy, which is supported by five arguments. First, post mortem studies have established that the noninfectious pulmonary complications of acute leukaemia include leukostasis, leukaemic infiltration and lysis pneumopathy [8], as well as alveolar proteinosis, which is extremely rare [37]. Secondly, opportunistic infections are not encountered at the earliest phase of AML [3]. In contrast to patients with acute lymphoid leukaemia, patients with AML FAB-M5 and infection usually have community-acquired bacteria. All our patients received combination antibiotic therapy for 7 days starting at ICU admission. However, we cannot strictly rule out the diagnosis of infection, based on our data including post mortem biopsies in only two historical controls. Along this line, CONFALONIERI et al. [38] reported benefits from steroids in patients with severe community-acquired pneumonia. Thirdly, our study was done in a homogeneous group of patients with AML FAB-M5 patients (monocytic subtype) and inaugural respiratory failure. Among the 20 historical controls, diffuse haemorrhage by BAL and post mortem biopsies was a major finding. We believe this finding is sufficiently suggestive to maintain a high level of suspicion for leukaemia-related pulmonary infiltrates in patients with untreated AML FAB-M5 and inaugural acute respiratory failure. However, overlap may occur between lysis pneumopathy and cytarabine-induced pulmonary toxicity. Such overlap would further support steroid therapy [39]. Fourthly, all patients underwent noninvasive diagnostic tests to rule out infection $[25,27]$. Also, all intubated patients underwent bronchoscopy and BAL, which consistently showed diffuse alveolar haemorrhage. Fifthly, the decrease in ICU mortality among dexamethasone patients in our study supports our presumptive diagnosis of leukaemia-related pulmonary involvement without infection. Lastly, changes in mortality between historical controls and steroid-treated cases could have been ascribable to differences in ICU management and ventilatory strategies. However, median tidal volume was $9(6-10) \mathrm{mL} \cdot \mathrm{kg}^{-1}$ in historical controls and 9 (7-10) $\mathrm{mL} \cdot \mathrm{kg}^{-1}$ in steroid-treated cases (p-value not significant). Corresponding figures for positive end-expiratory pressure were 7 (2-11) and 9 (5-15) $\mathrm{cmH}_{2} \mathrm{O}$ (p-value not significant).

In summary, adding dexamethasone to the chemotherapy regimen in AML FAB-M5 patients with acute respiratory failure from leukaemia-related pulmonary involvement significantly diminished ICU mortality. In addition, the rate of post-chemotherapy deterioration and the need for ventilatory support decreased with dexamethasone therapy. These results suggest that dexamethasone may be effective in decreasing leukaemic pulmonary infiltration and leukostasis and in preventing lysis pneumopathy. We found no increase in infection rates with dexamethasone therapy. Although these data are not sufficient to make a recommendation about using dexamethasone, they warrant a trial of dexamethasone in patients with AML FAB-M5 presenting as acute respiratory failure without evidence of infection.

\section{SUPPORT STATEMENT}

Supported by a grant from the Assistance-Publique Hôpitaux de Paris (AOM 04139) and the French Society for Intensive Care Medicine.

\section{STATEMENT OF INTEREST}

None declared.

\section{REFERENCES}

1 Chaoui $\mathrm{D}$, Legrand $\mathrm{O}$, Roche $\mathrm{N}$, et al. Incidence and prognostic value of respiratory events in acute leukemia. Leukemia 2004; 18: 670-675.

2 Puig N, De La Rubia J, Jarque I, et al. Characteristics of and risk factors for pneumonia in patients with hematological malignancies developing fever after autologous blood stem cell transplantation. Leuk Lymphoma 2007; 48: 2367-2374.

3 Azoulay E, Schlemmer B. Diagnostic strategy in cancer patients with acute respiratory failure. Intensive Care Med 2006; 32: 808-822. 
4 Azoulay E, Thiery G, Chevret S, et al. The prognosis of acute respiratory failure in critically ill cancer patients. Medicine (Baltimore) 2004; 83: 360-370.

5 Darmon M, Thiery G, Ciroldi M, et al. Intensive care in patients with newly diagnosed malignancies and a need for cancer chemotherapy. Crit Care Med 2005; 33: 2488-2493.

6 Potenza L, Luppi M, Morselli M, et al. Leukaemic pulmonary infiltrates in adult acute myeloid leukaemia: a high-resolution computerized tomography study. Br J Haematol 2003; 120: 1058-1061.

7 Thornton KA, Levis M. Images in clinical medicine. FLT3 mutation and acute myelogenous leukemia with leukostasis. $N$ Engl J Med 2007; 357: 1639.

8 Azoulay E, Fieux F, Moreau D, et al. Acute monocytic leukemia presenting as acute respiratory failure. Am J Respir Crit Care Med 2003; 167: 1329-1333.

9 Koh TT, Colby TV, Muller NL. Myeloid leukemias and lung involvement. Semin Respir Crit Care Med 2005; 26: 514-519.

10 Dutcher JP, Schiffer CA, Wiernik PH. Hyperleukocytosis in adult acute nonlymphocytic leukemia: impact on remission rate and duration, and survival. J Clin Oncol 1987; 5: 1364-1372.

11 Lester TJ, Johnson JW, Cuttner J. Pulmonary leukostasis as the single worst prognostic factor in patients with acute myelocytic leukemia and hyperleukocytosis. Am J Med 1985; 79: 43-48.

12 Ventura GJ, Hester JP, Smith TL, et al. Acute myeloblastic leukemia with hyperleukocytosis: risk factors for early mortality in induction. Am J Hematol 1988; 27: 34-37.

13 Lichtman MA, Rowe JM. Hyperleukocytic leukemias: rheological, clinical, and therapeutic considerations. Blood 1982; 60: 279-283.

14 Stucki A, Rivier AS, Gikic M, et al. Endothelial cell activation by myeloblasts: molecular mechanisms of leukostasis and leukemic cell dissemination. Blood 2001; 97: 2121-2129.

15 van Buchem MA, Colly LP, Hogendoorn PC, et al. Experimental myelocytic leukemia in the Brown-Norway rat as a model for pulmonary leukostasis. Am J Pathol 1991; 138: 777-780.

16 Prakash UB, Divertie MB, Banks PM. Aggressive therapy in acute respiratory failure from leukemic pulmonary infiltrates. Chest 1979; 75: 345-350.

17 Rossi GA, Balbi B, Risso M, et al. Acute myelomonocytic leukemia. Demonstration of pulmonary involvement by bronchoalveolar lavage. Chest 1985; 87: 259-260.

18 Valentino DJ 3rd, Gelfand MC, Nava VE, et al. Hypoxemic respiratory failure in a 57-year-old woman with acute monocytic leukemia. Chest 2005; 128: 3629-3633.

19 Luce JM, Montgomery AB, Marks JD, et al. Ineffectiveness of highdose methylprednisolone in preventing parenchymal lung injury and improving mortality in patients with septic shock. Am Rev Respir Dis 1988; 138: 62-68.

20 Bernard GR, Luce JM, Sprung CL, et al. High-dose corticosteroids in patients with the adult respiratory distress syndrome. $N$ Engl J Med 1987; 317: 1565-1570.

21 Steinberg KP, Hudson LD, Goodman RB, et al. Efficacy and safety of corticosteroids for persistent acute respiratory distress syndrome. N Engl J Med 2006; 354: 1671-1684.

22 Meduri GU, Headley AS, Golden E, et al. Effect of prolonged methylprednisolone therapy in unresolving acute respiratory distress syndrome: a randomized controlled trial. JAMA 1998; 280: 159-165.
23 De Botton S, Dombret H, Sanz M, et al. Incidence, clinical features, and outcome of all trans-retinoic acid syndrome in 413 cases of newly diagnosed acute promyelocytic leukemia. The European APL Group. Blood 1998; 92: 2712-2718.

24 Kelaidi C, Chevret S, De Botton S, et al. Improved outcome of acute promyelocytic leukemia with high WBC counts over the last 15 years: the European APL Group experience. J Clin Oncol 2009; 27: 2668-2676.

25 Azoulay E, Mokart D, Lambert J, et al. Diagnostic strategy for hematology and oncology patients with acute respiratory failure: randomized controlled trial. Am J Respir Crit Care Med 2010; 182 1038-1046.

26 Azoulay E, Alberti C, Bornstain C, et al. Improved survival in cancer patients requiring mechanical ventilatory support: impact of noninvasive mechanical ventilatory support. Crit Care Med 2001; 29: 519-525.

27 Azoulay E, Mokart D, Rabbat A, et al. Diagnostic bronchoscopy in hematology and oncology patients with acute respiratory failure: prospective multicenter data. Crit Care Med 2008; 36: 100-107.

28 Soares FA, Landell GA, Cardoso MC. Pulmonary leukostasis without hyperleukocytosis: a clinicopathologic study of 16 cases. Am J Hematol 1992; 40: 28-32.

29 McKee LC Jr, Collins RD. Intravascular leukocyte thrombi and aggregates as a cause of morbidity and mortality in leukemia. Medicine (Baltimore) 1974; 53: 463-478.

30 Tryka AF, Godleski JJ, Fanta CH. Leukemic cell lysis pneumonopathy. A complication of treated myeloblastic leukemia. Cancer 1982; 50: 2763-2770.

31 Dombret H, Hunault M, Faucher C, et al. Acute lysis pneumopathy after chemotherapy for acute myelomonocytic leukemia with abnormal marrow eosinophils. Cancer 1992; 69: 1356-1361.

32 Meduri GU, Tolley EA, Chinn A, et al. Procollagen types I and III aminoterminal propeptide levels during acute respiratory distress syndrome and in response to methylprednisolone treatment. Am J Respir Crit Care Med 1998; 158: 1432-1441.

33 Meduri GU, Golden E, Freire AX, et al. Methylprednisolone infusion in early severe ARDS: results of a randomized controlled trial. Chest 2007; 131: 954-963.

34 Inaba $\mathrm{H}$, Pui $\mathrm{CH}$. Glucocorticoid use in acute lymphoblastic leukaemia. Lancet Oncol 2010; 11: 1096-1106.

35 Ades L, Guerci A, Raffoux E, et al. Very long-term outcome of acute promyelocytic leukemia after treatment with all-trans retinoic acid and chemotherapy: the European APL Group experience. Blood 2010; 115: 1690-1696.

36 Schellongowski PST, Kundi M, Laczika K, et al. Prognostic factors for intensive care unit admission, intensive care outcome, and post-intensive care survival in patients with de novo acute myeloid leukemia: a single center experience. Haematologica 2011; 96: 23.

37 Cordonnier C, Fleury-Feith J, Escudier E, et al. Secondary alveolar proteinosis is a reversible cause of respiratory failure in leukemic patients. Am J Respir Crit Care Med 1994; 149: 788-794.

38 Confalonieri M, Urbino R, Potena A, et al. Hydrocortisone infusion for severe community-acquired pneumonia: a preliminary randomized study. Am J Respir Crit Care Med 2005; 171: 242-248.

39 Forghieri F, Luppi M, Morselli M, et al. Cytarabine-related lung infiltrates on high resolution computerized tomography: a possible complication with benign outcome in leukemic patients. Haematologica 2007; 92: e85-e90. 